\title{
УДАРНОЕ ВЗАИМОДЕЙСТВИЕ ЖИДКОСТИ И НАКЛОННОЙ ПЛАСТИНКИ, НАХОДЯЩЕЙСЯ НА ЕЕ СВОБОДНОЙ ПОВЕРХНОСТИ. ОПРЕДЕЛЕНИЕ СУММАРНЫХ СИЛОВЫХ ХАРАКТЕРИСТИК
}

Проведено комплексное поэтапное исследование задачи об ударном взаимодействии жидкости и наклонной пластинки, находящейся на ее свободной поверхности. При формулировке постановки задачи она сведена к краевой смешанной задаче для некоторой функции комплексного переменного, связанной с комплексным потенциалом течения, возникающего в результате ударного взаимодействия. Показано, что именно в такой постановке задача допускает аналитическое решение в виде квадратур. Выявлено, что полученные при этом интегралы в случае предельных переходов в окрестности особых точек точках стыка граничных условий различного типа - являются расходящимися и должны трактоваться в смысле конечной части по Адамару. На следующем этапе найдены положения зоны отрыва течения. Далее определение суммарных характеристик при ударном взаимодействии наклонной пластинки и жидкости сведено к вычислению полученных расходящихся интегралов с использованием формул Адамара - Манглера. При этом как положения зон инерционного отрыва течения, так и гидродинамические распределенные и суммарные характеристики пластинки найдены для широкого диапазона углов наклона пластинки к свободной поверхности жидкости. В данной части комплексной работы рассмотрены вопросы, связанные с определением коэффициентов присоединенных масс и моментов. Получены формулы для коэффициентов присоединенных масс и моментов в случае плоского течения. Представлены результаты расчетов коэффициентов присоединенных масс для горизонтального удара с вращением вертикальной пластины. Проведено сравнение значений коэффициентов присоединенных масс, полученных с помощью аналитического решения и с использованием интегралов в смысле конечной части по Адамару.

Ключевые слова: отрыв течения, удар, формулы Адамара - Манглера, наклонная пластинка, коэффициенты присоединенных масс.

Проведено комплексне поетапне дослідження задачі про ударну взаємодію рідини та похилої пластинки, яка знаходиться на її вільній поверхні. За формулюванням постановки задачі її зведено до крайової змішаної задачі для деякої функції комплексної змінної, що пов'язана із комплексним потенціалом течії, яка виникає внаслідок ударної взаємодії. Показано, що тільки в такій постановці задача має аналітичний розв'язок у вигляді квадратур. Виявлено, що отримані при цьому інтеграли у випадку граничних переходів в околі особливих точок точок стику граничних умов різного типу - виявляються розбіжними і повинні трактуватися в сенсі скінченної частини за Адамаром. На подальшому етапі знайдено розташування зони відриву течії. Далі визначення сумарних характеристик за ударної взаємодії похилої пластинки та рідини зведено до обчислення отриманих інтегралів, що розходяться, із застосуванням формул Адамара - Манглера. При цьому як положення зон інерційного відриву течії так і гідродинамічні розподілені й сумарні характеристики пластинки знайдено для широкого діапазону кутів нахилу пластинки до вільної поверхні рідини. В представленій частині комплексної роботи розглянуто питання, які пов'язані з визначенням коефіцієнтів приєднаних масс і моментів. Визначено основні формули для коефіцієнтів приєднаних мас i моментів у випадку плоскої течії. Наведено результати отриманих розрахунків коефіціснтів присднаних масс для горизонтального удару 3 рідиною 3 обертанням вертикальної пластинки. Проведено співставлення значень коефіціснтів присднаних мас, отриманих за допомогою аналітичного розв'язку та з використанням інтегралів у сенсі скінченної частини за Адамаром.

Ключові слова: відрив течії, удар, формули Адамара - Манглера, похила пластинка, коефіцієнти приєднаних мас.

(C) Гоман О.Г., Катан В.А., 2018 
Difficult research of the problem of impact interaction of fluid and an inclined plate which located on its free surface is carried out. The main formulation of the problem is reduced to a boundary value problem for a some function of complex variable associated with a complex potential of the flow due to impact interaction. It was noticed that only formulation of problem admits an analytical solution in the form of the quadrature. The fact was observed that obtaining integrals are divergent in the case of the limit in the neighborhood of singular points - junction points of the boundary conditions of various types - and must be interpreted in the sense of Hadamard finite part. On the other stage of solution the positions of separation zones were found. Than the determining of summaries characteristics of impact interaction of fluid and inclined plate is reduced to calculating of the integrals which are divergenting using formulas Hadamard - Mangler. In the wide range of angles for inclined plate the position of separation zones and distributing gidrodynamic and summaries characteristics of the plate were found. This part of complex research is devoted to questions are connected with determining of added mass' and moments' coefficients. The formulas of added mass' and moments' coefficients are obtained for the case with two-dimensional flow. The numerical results of addition mass' and moments' coefficients for the case of gorizontal impact with rotation of vertical plate are presented. The comparison between values of added mass' and moments' coefficients were obtained by analitical solution and by obtaining integrals in the sense of Hadamard finite part is considered.

Key words: separation of flow, impact, Hadamard - Mangler formulas, inclined plate, added mass coefficients.

Введение. Улучшение эксплуатационных характеристик высокоскоростных гидродинамических аппаратов, в частности, их рулевых устройств, невозможно без детального исследования и решения задач гидродинамического ударного взаимодействия. Данная статья является продолжением работ авторов [1; 2], в которых изложена математическая постановка, общий подход метода решения задачи, получено аналитическое решение, определены положения зоны отрыва течения, а также распределенные силовые характеристики, в частности, распределение импульсивного давления поверхности пластинки.

Постановка задачи. Пусть наклонная пластина ширины $b$ плавает под углом $\alpha \pi$ на свободной поверхности несжимаемой идеальной жидкости, находящейся в покое и занимающей полупространство (рис.1).

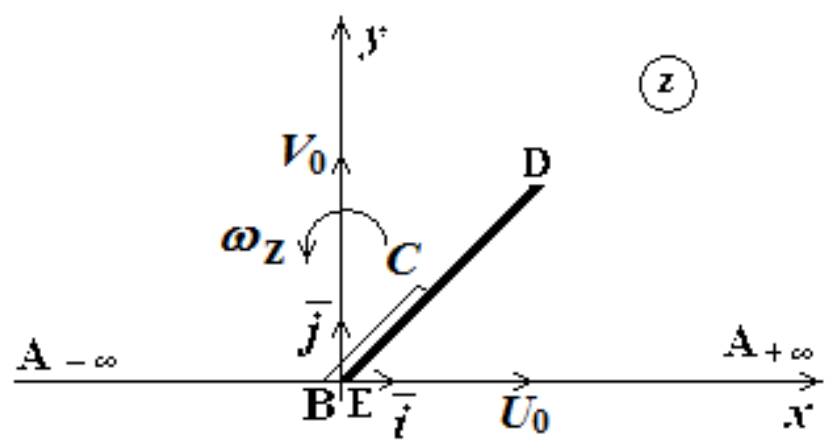

Рис. 1. Область решения задачи с учетом зоны отрыва течения

Ось Оу декартовой системы координат, как обычно, направим по нормали к невозмущенной свободной поверхности жидкости внутрь последней, а ось О $x-$ вдоль свободной поверхности. Предполагается, что ударные импульсы подействовали так, что в результате удара элементы пластинки приобретают скорость

$$
\bar{V}=\left(U_{0}-\omega_{z} y\right) \vec{i}+\left(V_{0}+\omega_{z} x\right) \vec{j}
$$

где $U_{0}$ - поступательная скорость вдоль оси $\mathrm{O} x, V_{0}$ - поступательная скорость 
вдоль оси О $y, \omega_{z}$ - угловая скорость. На схеме к рассматриваемой задаче, показанной на рис. 1 , жидкость занимает верхнюю полуплоскость $y \geq 0$.

В предположении о наличии отрыва $B C$ условие безотрывности обтекания распространяется только на участке контура $C D E$, причем положение крайней точки отрыва $C$ заранее неизвестно, и имеет вид

$$
\left.\frac{\partial \varphi}{\partial n}\right|_{C D E}=\bar{V} \cdot \bar{n} .
$$

На участке $C D$ имеем следующее выражение:

$$
\left.\frac{\partial \varphi}{\partial n}\right|_{C D}=\bar{V} \cdot \bar{n}_{r}=V_{n_{r}}=-U_{0} \sin \alpha \pi+V_{0} \cos \alpha \pi+\omega_{z}(x \cos \alpha \pi+y \sin \alpha \pi),
$$

а на участке $D E$ выражение вида:

$$
\left.\frac{\partial \varphi}{\partial n}\right|_{D E}=\bar{V} \cdot \bar{n}_{f}=V_{n_{f}}=U_{0} \sin \alpha \pi-V_{0} \cos \alpha \pi-\omega_{z}(x \cos \alpha \pi+y \sin \alpha \pi) .
$$

На свободной границе - оси О $x$ и участке отрыва $B C$ - имеем в качестве условия равенство нулю импульсивного давления, то есть соотношение

$$
\varphi=0 \text {. }
$$

Следовательно, имеем смешанную задачу для гармонической функции потенциала скоростей $\varphi$.

На участке безотрывного обтекания $E D C$, как и ранее [1;2], для функции тока $\psi$ с точностью до несущественной константы, имеем следующее выражение:

$$
\psi=U_{0} y-V_{0} x-\frac{\omega_{z}}{2}\left(x^{2}+y^{2}\right) .
$$

Таким образом, в комплексной области $z=x+i y$ для функции

$$
\chi=-i w=\psi-i \varphi
$$

получаем задачу Келдыша-Седова [3]: на границе $C D E$ задана ее действительная часть вида:

$$
\left.\operatorname{Re} \chi\right|_{C D E}=U_{0} y-V_{0} x-\frac{\omega_{z}}{2}\left(x^{2}+y^{2}\right),
$$

а на границах $A_{-\infty} B, B C, E A_{+\infty}$ известна ее мнимая часть, заданная соотношениями:

$$
\left.\operatorname{Im} \chi\right|_{A_{-\infty} B}=0,\left.\operatorname{Im} \chi\right|_{B C}=0,\left.\operatorname{Im} \chi\right|_{E A_{+\infty}}=0 .
$$

Аналитическое исследование. Для произвольного угла наклона пластинки решение поставленной задачи $\chi(t)$ определяется формулой [1]:

$$
\chi(t)=\psi(t)-i \varphi(t)=
$$

$=-\frac{1}{\pi} \sqrt{(t+q)(t-1)}\left(\frac{\alpha}{1-\alpha}\right)^{1-\alpha}\left[b\left(U_{0} \sin \alpha \pi-V_{0} \cos \alpha \pi\right) J_{1}(t)-\frac{\omega_{z} b^{2}}{2}\left(\frac{\alpha}{1-\alpha}\right)^{1-\alpha} J_{2}(t)\right]$, (1),

где $t$ - параметрическая переменная; решение содержит неизвестный параметр $q$. 
В предположении о наличии только одной зоны отрыва, ее положение находится по принципу Огазо [1] с использованием сингулярных интегралов в смысле Адамара $[4 ; 5]$, и в данной работе полагаем, что значение координаты $q$ известно как функция от значения кинематического параметра $\Lambda=\frac{\omega_{z} b}{U_{n}}$, сформированного по нормальной скорости $U_{n}$, в известном для каждого угла $\alpha \pi$ диапазоне его изменения [1].

Вычисление коэффициентов присоединенных масс. Для определения присоединенных масс вводим единичные потенциалы

$$
\varphi=U_{0} \varphi_{1}+V_{0} \varphi_{2}+\omega_{z} \varphi_{6}
$$

и соответствующие им функции тока

$$
\psi=U_{0} \psi_{1}+V_{0} \psi_{2}+\omega_{z} \psi_{6} .
$$

Значения единичных потенциалов на смоченной части пластины $\left(-q<\xi_{0}<1\right)$ : определяются согласно формуле (1) и равны:

$$
\begin{gathered}
\varphi_{1}\left(\xi_{0}\right)=\frac{b \sin \alpha \pi}{\pi}\left(\frac{\alpha}{1-\alpha}\right)^{1-\alpha} \bar{\varphi}_{1}\left(\xi_{0}\right), \\
\varphi_{2}\left(\xi_{0}\right)=-\frac{b \cos \alpha \pi}{\pi}\left(\frac{\alpha}{1-\alpha}\right)^{1-\alpha} \bar{\varphi}_{1}\left(\xi_{0}\right), \\
\varphi_{3}\left(\xi_{0}\right)=-\frac{1}{\pi}\left(\frac{\alpha}{1-\alpha}\right)^{2(1-\alpha)} \frac{b^{2}}{2} \bar{\varphi}_{3}\left(\xi_{0}\right),
\end{gathered}
$$

а значения единичных функций тока на контуре равны:

$$
\begin{aligned}
\psi_{1}\left(\xi_{0}\right) & =\left(\frac{\alpha}{1-\alpha}\right)^{1-\alpha} b \sin \alpha \pi \bar{\psi}_{1}\left(\xi_{0}\right), \\
\psi_{2}\left(\xi_{0}\right) & =-\left(\frac{\alpha}{1-\alpha}\right)^{1-\alpha} b \cos \alpha \pi \bar{\psi}_{1}\left(\xi_{0}\right), \\
\psi_{3}\left(\xi_{0}\right) & =-\left(\frac{\alpha}{1-\alpha}\right)^{2(1-\alpha)} \frac{b^{2}}{2}\left(\bar{\psi}_{1}\left(\xi_{0}\right)\right)^{2},
\end{aligned}
$$

где введены дополнительные обозначения

$$
\begin{gathered}
\bar{\varphi}_{1}\left(\xi_{0}\right)=\sqrt{\left(\xi_{0}+q\right)\left(1-\xi_{0}\right)} J_{1}\left(\xi_{0}\right), \bar{\varphi}_{3}\left(\xi_{0}\right)=\sqrt{\left(\xi_{0}+q\right)\left(1-\xi_{0}\right)} J_{2}\left(\xi_{0}\right), \\
\bar{\psi}_{1}\left(\xi_{0}\right)=\left(\xi_{0}+\frac{1-\alpha}{\alpha}\right)^{1-\alpha}\left(1-\xi_{0}\right)^{\alpha} .
\end{gathered}
$$

Формулы для присоединенных масс в случае плоского течения имеют вид

$$
\lambda_{i k}=-\rho \int_{C} \varphi_{i} d \psi_{k}, \quad i, k=\overline{1,3},
$$

причем контур $C$ обходится против движения часовой стрелки. Интегрирование можно проводить как в физической плоскости $z$, так и в плоскости параметрической переменной $t$. В плоскости $t$ контур $C$ будет представлять собой 
верхний берег разреза вдоль отрезка действительной оси $\xi \in[-q, 1]$ от точки $E^{\prime}\left(\xi_{E}=1\right)$ до точки $C^{\prime}\left(\xi_{C}=-q\right)$, так что формулы (11) с учетом направления интегрирования принимают вид

$$
\lambda_{i k}=\rho \int_{-q}^{1} \varphi_{i} d \psi_{k}, \quad i, k=\overline{1,3} .
$$

После ввода соотношений для коэффициентов присоединенных масс в виде

$$
\bar{\lambda}_{11}=\frac{\lambda_{11}}{\rho b^{2}}, \quad \bar{\lambda}_{12}=\frac{\lambda_{12}}{\rho b^{2}}, \quad \bar{\lambda}_{22}=\frac{\lambda_{22}}{\rho b^{2}}, \quad \bar{\lambda}_{16}=\frac{\lambda_{16}}{\rho b^{3}}, \quad \bar{\lambda}_{26}=\frac{\lambda_{26}}{\rho b^{3}}, \quad \bar{\lambda}_{66}=\frac{\lambda_{66}}{\rho b^{4}},
$$

последовательно получаем выражения:

$$
\begin{gathered}
\bar{\lambda}_{11}=\frac{1}{\pi}\left(\frac{\alpha}{1-\alpha}\right)^{2(1-\alpha)} \sin ^{2} \alpha \pi \int_{-q}^{1} \bar{\varphi}_{1}\left(\xi_{0}\right) d \bar{\psi}_{1}\left(\xi_{0}\right), \\
\bar{\lambda}_{12}=-\frac{1}{\pi}\left(\frac{\alpha}{1-\alpha}\right)^{2(1-\alpha)} \sin \alpha \pi \cos \alpha \pi \int_{-q}^{1} \bar{\varphi}_{1}\left(\xi_{0}\right) d \bar{\psi}_{1}\left(\xi_{0}\right), \\
\bar{\lambda}_{16}=-\frac{1}{\pi}\left(\frac{\alpha}{1-\alpha}\right)^{3(1-\alpha)} \sin \alpha \pi \int_{-q}^{1} \bar{\varphi}_{1}\left(\xi_{0}\right) \bar{\psi}_{1}\left(\xi_{0}\right) d \bar{\psi}_{1}\left(\xi_{0}\right), \\
\bar{\lambda}_{22}=\frac{1}{\pi}\left(\frac{\alpha}{1-\alpha}\right)^{2(1-\alpha)} \cos ^{2} \alpha \pi \int_{-q}^{1} \bar{\varphi}_{1}\left(\xi_{0}\right) d \bar{\psi}_{1}\left(\xi_{0}\right), \\
\bar{\lambda}_{26}=\frac{1}{\pi}\left(\frac{\alpha}{1-\alpha}\right)^{3(1-\alpha)} \cos \alpha \pi \int_{-q}^{1} \bar{\varphi}_{1}\left(\xi_{0}\right) \bar{\psi}_{1}\left(\xi_{0}\right) d \bar{\psi}_{1}\left(\xi_{0}\right), \\
\bar{\lambda}_{66}=\frac{1}{2 \pi}\left(\frac{\alpha}{1-\alpha}\right)^{4(1-\alpha)} \int_{-q}^{1} \bar{\varphi}_{3}\left(\xi_{0}\right) \bar{\Psi}_{1}\left(\xi_{0}\right) d \bar{\psi}_{1}\left(\xi_{0}\right) .
\end{gathered}
$$

Таким образом, расчет коэффициентов присоединенных масс сводится к вычислению трех интегралов, а именно:

$$
\begin{gathered}
I_{11}=\int_{-q}^{1} \bar{\varphi}_{1}\left(\xi_{0}\right) d \bar{\psi}_{1}\left(\xi_{0}\right), \quad I_{111}=\int_{-q}^{1} \bar{\varphi}_{1}\left(\xi_{0}\right) \bar{\psi}_{1}\left(\xi_{0}\right) d \bar{\psi}_{1}\left(\xi_{0}\right), \\
I_{311}=\int_{-q}^{1} \bar{\varphi}_{3}\left(\xi_{0}\right) \bar{\psi}_{1}\left(\xi_{0}\right) d \bar{\psi}_{1}\left(\xi_{0}\right) .
\end{gathered}
$$

Поскольку имеется выражение 


$$
d \bar{\psi}_{1}\left(\xi_{0}\right)=\bar{\psi}_{1}\left(\xi_{0}\right)\left[\frac{1-\alpha}{\xi_{0}+\frac{1-\alpha}{\alpha}}-\frac{\alpha}{1-\xi_{0}}\right] d \xi_{0}=-\frac{\xi_{0} \bar{\psi}_{1}\left(\xi_{0}\right) d \xi_{0}}{\left(\xi_{0}+\frac{1-\alpha}{\alpha}\right)\left(1-\xi_{0}\right)},
$$

то для интегралов (13) получим представления в следующих формулах

$$
\begin{gathered}
I_{11}=-\int_{-q}^{1} \xi_{0}\left(\xi_{0}+q\right)^{\frac{1}{2}}\left(\xi_{0}+\frac{1-\alpha}{\alpha}\right)^{-\alpha}\left(1-\xi_{0}\right)^{\alpha-\frac{1}{2}} J_{1}\left(\xi_{0}\right) d \xi_{0}, \\
I_{111}=-\int_{-q}^{1} \xi_{0}\left(\xi_{0}+q\right)^{\frac{1}{2}}\left(\xi_{0}+\frac{1-\alpha}{\alpha}\right)^{1-2 \alpha}\left(1-\xi_{0}\right)^{2 \alpha-\frac{1}{2}} J_{1}\left(\xi_{0}\right) d \xi_{0}, \\
I_{311}=-\int_{-q}^{1} \xi_{0}\left(\xi_{0}+q\right)^{\frac{1}{2}}\left(\xi_{0}+\frac{1-\alpha}{\alpha}\right)^{1-2 \alpha}\left(1-\xi_{0}\right)^{2 \alpha-\frac{1}{2}} J_{2}\left(\xi_{0}\right) d \xi_{0} .
\end{gathered}
$$

В этих представлениях два последних интеграла являются несобственными с особой точкой $\xi_{0}=1$ в диапазоне $\alpha \in(0 ; 0,25)$, а для интеграла $I_{11}-$ в диапазоне $\alpha \in(0 ; 0,5)$. Следовательно, при указанных значениях параметра $\alpha$ особую точку можно отделять окрестностью размера $\varepsilon$ и интегрировать по ней с учетом разложения подынтегральных функций. Следовательно, окончательные формулы для вспомогательных интегралов (14) - (16) при этом будут иметь вид

$$
\begin{aligned}
& I_{11}=-\int_{-q}^{1-\varepsilon} \xi_{0}\left(\xi_{0}+q\right)^{\frac{1}{2}}\left(\xi_{0}+\frac{1-\alpha}{\alpha}\right)^{-\alpha}\left(1-\xi_{0}\right)^{\alpha-\frac{1}{2}} J_{1}\left(\xi_{0}\right) d \xi_{0}-2 \alpha^{\alpha} \sqrt{1+q} J_{1}(1) \frac{\varepsilon^{\alpha+\frac{1}{2}}}{2 \alpha+1}, \\
& I_{111}=-\int_{-q}^{1-\varepsilon} \xi_{0}\left(\xi_{0}+q\right)^{\frac{1}{2}}\left(\xi_{0}+\frac{1-\alpha}{\alpha}\right)^{1-2 \alpha}\left(1-\xi_{0}\right)^{2 \alpha-\frac{1}{2}} J_{1}\left(\xi_{0}\right) d \xi_{0}-\frac{2 \sqrt{1+q}}{\alpha^{1-2 \alpha}} J_{1}(1) \frac{\varepsilon^{2 \alpha+\frac{1}{2}}}{4 \alpha+1},(17) \\
& I_{311}=-\int_{-q}^{1-\varepsilon} \xi_{0}\left(\xi_{0}+q\right)^{\frac{1}{2}}\left(\xi_{0}+\frac{1-\alpha}{\alpha}\right)^{1-2 \alpha}\left(1-\xi_{0}\right)^{2 \alpha-\frac{1}{2}} J_{2}\left(\xi_{0}\right) d \xi_{0}-\frac{2 \sqrt{1+q}}{\alpha^{1-2 \alpha}} J_{2}(1) \frac{\varepsilon^{2 \alpha+\frac{1}{2}}}{4 \alpha+1} .
\end{aligned}
$$

Интегрирование в присутствующих здесь собственных интегралах может быть осуществлено численно по любой подходящей квадратурной формуле, при этом, как уже указывалось, значения интегралов $J_{i}\left(\xi_{0}\right), i=\overline{1,2}$, во внутренних узлах квадратурной формулы вычисляются согласно описанной выше процедуре вычисления интегралов в смысле Коши, а их значения в крайних точках $\xi_{0}=-q$ и $\xi_{0}=1-$ по формулам Адамара - Манглера. 
В частных случаях, когда $\alpha=0,5$ (при этом $\left.J_{1}\left(\xi_{0}\right)\right|_{\alpha=0,5} \rightarrow \infty$ при $\xi_{0} \rightarrow 1$ ) и $\alpha=0,25$ (при этом $\left.J_{2}\left(\xi_{0}\right)\right|_{\alpha=0,25} \rightarrow \infty$ при $\xi_{0} \rightarrow 1$ ), вместо выражений (14) - (16) получены интегралы вида

$$
\begin{aligned}
& I_{11}=-\int_{-q}^{1} \xi_{0}\left(\xi_{0}+q\right)_{2}^{\frac{1}{2}}\left(\xi_{0}+1\right)^{-\frac{1}{2}} J_{1}\left(\xi_{0}\right) d \xi_{0}, \\
& I_{111}=-\int_{-q}^{1} \xi_{0}\left(\xi_{0}+q\right)^{\frac{1}{2}}\left(1-\xi_{0}\right)^{\frac{1}{2}} J_{1}\left(\xi_{0}\right) d \xi_{0}, \\
& I_{311}=-\int_{-q}^{1} \xi_{0}\left(\xi_{0}+q\right)^{\frac{1}{2}}\left(\xi_{0}+3\right)^{\frac{1}{2}} J_{2}\left(\xi_{0}\right) d \xi_{0} .
\end{aligned}
$$

Указанные в (18) интегралы $I_{11}$ и $I_{311}$ имеют логарифмическую особенность при $\xi_{0} \rightarrow 1$ за счет наличия в них функций $J_{1}\left(\xi_{0}\right)$ и $J_{2}\left(\xi_{0}\right)$ (интеграл $I_{111}$ такой особенности не имеет за счет наличия множителя $\left(1-\xi_{0}\right) \frac{1}{2}$ при $\left.J_{1}\left(\xi_{0}\right)\right)$, так что отделяя в этих интегралах точку $\xi_{0}=1$ окрестностью размером $\varepsilon$ и пользуясь выражениями для $\left.J_{1}\left(\xi_{0}\right)\right|_{\alpha=0,5}$ и $\left.J_{2}\left(\xi_{0}\right)\right|_{\alpha=0,25}$ из [2], можно получить формулы, аналогичные (17). Вывод этих формул остается за рамками данной статьи.

По описанному алгоритму были проведены расчеты коэффициентов присоединенных масс пластинки для углов ее наклона $\alpha=90^{0}, 60^{\circ}, 45^{0}, 30^{\circ}, 10^{0}$ и $1^{0}$ при предельном значении кинематического параметра $\Lambda_{\text {пред }}$ и при значениях $\Lambda=0,5,0,-1,-2,-100$.

Результаты расчетов коэффициентов присоединенных масс для горизонтального удара с вращением вертикальной пластины, полученные с помощью аналитического решения и с использованием интегралов в смысле конечной части по Адамару, представлены в табл. 1.

Таблица 1

Сравнение значений коэффициентов присоединенных масс для горизонтального удара с вращением вертикальной пластинки $\left(\alpha=90^{0}\right)$

\begin{tabular}{|c|c|c|c|c|c|c|c|}
\hline$\Lambda$ & 1,3264 & 1,0000 & 0,5000 & 0 & $-1,0000$ & $-2,0000$ & $-100,0$ \\
\hline $\bar{\lambda}_{11} *$ & 0,3779 & 0,3546 & 0,3527 & 0,3528 & 0,3529 & 0,3530 & 0,3530 \\
\hline $\bar{\lambda}_{11} * *$ & 0,3839 & 0,3595 & 0,3570 & 0,3570 & 0,3570 & 0,3570 & 0,3569 \\
\hline $\bar{\lambda}_{16} *$ & $-0,2170$ & $-0,1968$ & $-0,1941$ & $-0,1940$ & $-0,1940$ & $-0,1940$ & $-0,1940$ \\
\hline $\bar{\lambda}_{16} * *$ & $-0,2163$ & $-0,1958$ & $-0,1931$ & $-0,1931$ & $-0,1931$ & $-0,1931$ & $-0,1931$ \\
\hline $\bar{\lambda}_{66} *$ & 0,1372 & 0,1201 & 0,1170 & 0,1169 & 0,1168 & 0,1168 & 0,1168 \\
\hline $\bar{\lambda}_{66} * *$ & 0,1368 & 0,1197 & 0,1165 & 0,1164 & 0,1164 & 0,1164 & 0,1164 \\
\hline
\end{tabular}

* - расчет с использованием интегралов в смысле конечной части по Адамару;

** - расчет согласно аналитическому решению. 
Следует отметить, что совпадение значений коэффициентов присоединенных масс хорошее. При этом относительная погрешность определения коэффициента присоединенной массы $\lambda_{11}$ составила не больше $1,5 \%$, а для коэффициентов $\lambda_{16}$ и $\lambda_{66}$ - не больше $0,5 \%$. Численное интегрирование при вычислениях интегралов $J_{k}\left(\xi_{0}\right), \quad k=1,2$ в приведенных выше формулах выполнялось по адаптивной процедуре Симпсона, а для малых значений углов наклона пластинки $\alpha=10^{0}$ и $\alpha=1^{0}$ применялась квадратурная формула Ромберга [6].

Как показывают расчетные данные, основное влияние на значение любого из коэффициентов присоединенной массы оказывает угол наклона пластинки; изменение кинематического параметра $\Lambda$ приводит к изменению этих коэффициентов не более чем на $15 \%$.

Выводы. Хорошее совпадение данных аналитического решения и решения, полученного через интегралы в смысле Адамара, позволяет сделать вывод о применимости данного подхода к определению коэффициентов присоединенных масс и моментов при ударном взаимодействии твердого тела и жидкости с образованием одной зоны инерционного отрыва.

\section{Библиографические ссылки}

1. Гоман, О.Г. Ударное взаимодействие жидкости и наклонной пластинки, находящейся на ее свободной поверхности. Определение положения зоны отрыва [Текст]/ О.Г. Гоман, В.А. Катан // Вісн. ДНУ. Сер.: Механіка. - 2016. - № 5(24). - Вип. 20. C. $28-38$.

2. Гоман, О.Г. Ударное взаимодействие жидкости и наклонной пластинки, находящейся на ее свободной поверхности. Определение распределенных силовых характеристик [Текст]/ О.Г. Гоман, В.А. Катан // Вісн. ДНУ. Сер.: Механіка. - 2017. № 5(25). - Вип. 21. - С. $58-65$.

3. Мусхелишвили, Н. И. Некоторые основные задачи математической теории упругости [Текст] / Н. И. Мусхелишвили. - М.: Наука,1966. - 707c.

4. Общая теория аэродинамики больших скоростей под редакцией [Текст]/ под ред. У.Р. Сирса. - М.: Воениздат, 1962. - 300 с.

5. Адамар, Ж. Задача Коши для линейных уравнений с частными производными гиперболического типа [Текст]/ Ж. Адамар. - М.: Наука, 1978. - 352 с.

6. Мэтьюз Джон Г. Численные методы. Использование MATLAB [Tекст]/ Джон Г. Мэтьюз, Куртис Д. Финк. 3-е изд.- М., 2001. - 720 с.

Надійшла до редколегії 25.05.18. 